\title{
THE EFFECT OF CLIPPING IN FLUORESCENCE CORRELATION SPECTROSCOPY
}

The effect of clipping, the usual operation in the photon correlation spectroscopy, is discussed if used in the fluorescence correlation spectroscopy. The distortion of the autocorrelation function caused by the clipping operation is shown to be linear and the signal-to-noise ratio reduced $(\pi / 2)^{1 / 2}$ times.

\section{Introduction}

According to the fluctuation-dissipation theorem [ $\left.{ }^{1}\right]$, the same kinetic parameters that describe the relaxation of the system after a macroscopic perturbation, also describe the dynamics of spontaneous fluctuations about equilibrium. This is the theoretical foundation of concentration correlation spectroscopy - a relatively new method for the study of chemical kinetics and diffusion processes. Unlike the various methods which use a macroscopic perturbation of the system for obtaining its relaxation towards equilibrium (e. g., jump methods, sound absorption, etc.), correlation spectroscopy makes it possible to determine the same kinetic parameters through the autocorrelation function of concentration fluctuations at thermodynamic equilibrium. To obtain the concentration fluctuations, we need physical probes for continuous monitoring of the concentrations of all relevant chemical components. A convenient and sensitive means for the measurement of instantaneous concentrations of certain molecules is fluorescence $\left[{ }^{2-4}\right]$. In the first experiment in this field, the fluorescence correlation spectroscopy (FCS), carried out by Magde, Elson and Webb [ ${ }^{3}$, the photomultiplier current was measured, its autocorrelation function was computed and the kinetic parameters of chemical reactions and those of diffusion were obtained.

The autocorrelation function of the light intensity $I(t)$ can be defined as

$$
g^{(2)}(\tau)=\langle I(t) \quad I(t+\tau)\rangle,
$$

the angle brackets denoting an average over an ensemble of samples or, alternatively, for an ergodic field, a time average.

Note that the light intensity fluctuations can be caused not only by power fluctuations in the light source (which is the object in FCS, where the information about the dynamics of fluorescent molecules is obtained), but also by the interference effects. The latter are the study objects in the so-called photon correlation spectroscopy (PCS) $\left[{ }^{5}\right]$ and express information about the light intensity spectrum (i. e., about the molecular emission / scattering process). We recall PCS here because its experiment 
is based on the modern photon counting method which has been highly developed in recent years.

In a typical PCS experiment the autocorrelation function of the photon counting fluctuations is measured:

$$
\hat{g}^{(2)}(\tau)=\langle n(\tau) n(t+\tau)\rangle,
$$

where $n(t)$ is the number of photons counted during the time interval $(t, t+T)$. In order to increase the time resolution and to reduce the apparatus complexity, a clipped autocorrelation function is often measured $\left[{ }^{6}\right]$ :

Here

$$
\hat{g}_{c}(\tau)=\left\langle n_{c}(t) n(t+\tau)\right\rangle \text {. }
$$

$$
\begin{array}{rlll}
n_{c}(t)=1 & \text { if } & & n(t)>k, \\
=0 & \text { if } & & n(t) \leqslant k
\end{array}
$$

and $k$ is the clipping level.

In PCS in the case of thermal light with an arbitrary spectrum, a linear relation between $\hat{g}^{(2)}(\tau)$ and $\hat{g}_{c}(\tau)$ holds $\left[{ }^{6,7}\right]$. The assumptions used in deriving this relation in PCS $\left[{ }^{7}\right]$ do not hold in FCS where the spatial correlation properties and the relations between the first and second order correlation functions are different. Since in the FCS experiment significantly greater counting rates are required $\left[{ }^{4}\right]$ than those in the PCS experiment, one needs simplifications in the FCS apparatus even more. In this paper we examine how the relaxation processes, as studied by the FCS method, are expressed by the clipped autocorrelation function, and what effect the clipping operation has upon the accuracy of the determination of kinetic parameters (in the case of intense fluorescence $\langle n\rangle \gg 1)$. An expression for the clipped autocorrelation function of the fluorescence photon count is found, and the signal-to-noise ratio in the FCS experiment is given.

\section{The clipped autocorrelation function of the light intensity}

In the photon correlation spectroscopy of optical fields, having a Gaussian-distributed complex electric field amplitude (called Gaussian light), a useful quantity is the conditional expectation $E\left\{I(\tau) \mid I(0)=I_{0}\right\}$ of observing an intensity $I(\tau)$ at time $\tau$, given the intensity $I_{0}$ at time zero. For this quantity the following relation holds $\left.{ }^{8}\right]$ :

$$
E\left\{I(\tau) \mid I(0)=I_{0}\right\}=\left(I_{0}-\langle I\rangle\right) f(\tau)+\langle I\rangle,
$$

where $\langle I\rangle$ is the nonzero average intensity and $f(\tau)$ is a function of the delay time $\tau$. In the Appendix we have shown that the same relation also holds for the fluorescence intensity in FCS (in the case $\beta \equiv \operatorname{var} I /\langle I\rangle^{2} \ll 1$; see (A9)).

In practice, as we measure during the nonzero counting intervals $T$, we obtain the integral intensities $U(\tau)$ rather than the instantaneous intensities $I(\tau)$.

$$
U(\tau)=\int_{\tau}^{\tau+T} I(t) \mathrm{d} t
$$

For the conditional expectation value of the integral intensity, a relation analogous to (4) holds: 


$$
E\left\{U(\tau) \mid U(0)=U_{0}\right\}=\left(U_{0}-\langle U\rangle\right) f(\tau)+\langle U\rangle .
$$

For the estimate of the autocorrelation function $\hat{g}^{(2)}(\tau)=\langle n(0) n(\tau)\rangle$, we obtain

$$
\begin{gathered}
E\{n(0) n(\tau)\}=\int E\left\{n(0) \mid U(0)=U_{0}\right\} E\left\{n(\tau) \mid U(0)=U_{0}\right\} w\left(U_{0}\right) \mathrm{d} U_{0}= \\
=\alpha^{2}\left[f(\tau) \operatorname{var} U+\langle U\rangle^{2}\right] .
\end{gathered}
$$

Here $w(U)$ is the integral intensity distribution and $\alpha$ is the counting efficiency:

$$
\alpha=U^{-1} E(n \mid U) .
$$

As the autocorrelation function can be expressed as follows:

$$
g^{(2)}(\tau)=f(\tau) \operatorname{var} I+\langle I\rangle^{2},
$$

the estimate $\hat{g}^{(2)}(\tau)$ is linearly distorted for finite values of $T$.

For an estimate of the clipped autocorrelation function we obtain:

$$
\begin{gathered}
E\left\{n_{c}(0) n(\tau)\right\}=\int E\left\{n_{c}(0) \mid U(0)=U_{0}\right\} E\left\{n(\tau) \mid U(0)=U_{0}\right\} \omega\left(U_{0}\right) \mathrm{d} U_{0}= \\
=\alpha\left[f(\tau) \operatorname{cov}\left(n_{c}, U\right)+\left\langle n_{c}\right\rangle\langle U\rangle\right] .
\end{gathered}
$$

It follows from the equations (7) and (10) that a linear relation between the estimates of the clipped and nonclipped autocorrelation functions holds. Thus, the same information about the autocorrelation function of the light intensity is contained in both of them.

\section{The signal-to-noise ratio of the clipped autocorrelation function in FCS}

The clipped autocorrelation function is expressed in (10). Let us estimate the quantity

$$
E\left\{n_{c}(0) \mid U(0)=U_{0}\right\}=\sum_{n=k+1}^{\infty} p\left(n \mid U_{0}\right) .
$$

The distribution $p\left(n \mid U_{0}\right)$ of the count of $n$ photons, if the integral intensity is $U_{0}$, is Poissonian with a mean $E\left\{n \mid U_{0}\right\}=\alpha U_{0}$.

If the condition $\langle n\rangle \gg 1$ holds, we can integrate over a Gaussian distribution instead of the summation in (11):

$$
\begin{aligned}
E\left\{n_{c}(0) \mid U(0)=U_{0}\right\}= & \frac{1}{\left[2 \pi \operatorname{var} n\left(U_{0}\right)\right]^{1 / 2}} \int_{k}^{\infty} \exp \left\{-\frac{\left(x-\alpha U_{0}\right)}{2\left[\operatorname{var} n\left(U_{0}\right)\right]^{1 / 2}}\right\} d x= \\
& =\frac{1}{2}\left\{1-\Phi\left(\frac{k-\alpha U_{0}}{\left(\alpha U_{0}\right)^{1 / 2}}\right)\right\}
\end{aligned}
$$

where $\Phi(x)$ is the Gaussian probability integral. If $\langle n\rangle \gg 1$ (i.e., $\left.\alpha U_{0} \gg 1\right)$, the argument of the function $\Phi(x)$ is much less than unity, so that

$$
E\left\{n_{c}(0) \mid U(0)=U_{0}\right\} \simeq\left\langle n_{c}\right\rangle+\left.\frac{1}{2} \frac{\alpha U_{0}-\langle n\rangle}{\left(\alpha U_{0}\right)^{1 / 2}} \cdot \frac{\mathrm{d} \Phi(x)}{\mathrm{d} x}\right|_{\substack{x=\underbrace{k-\langle n\rangle}_{\left(\alpha U_{0}\right)^{1 / 2}} \\(6)}} \cdot
$$

Using the expressions (6) and (13), we obtain for the clipped autocorrelation function (10) 


$$
E\left\{n_{c}(0) n(\tau)\right\} \simeq\left\langle n_{c}\right\rangle\langle n\rangle+\left.\frac{1}{2} \frac{\mathrm{d} \Phi(x)}{\mathrm{d} x}\right|_{x=\sum_{x-\langle n\rangle}^{\langle n\rangle^{1 / 2}}} \beta f(\tau)\langle n\rangle^{3 / 2} .
$$

Consider now the FCS experiment carried out according to the following scheme. The fluorescence intensity measurement is executed at $M+R$ consecutive counting intervals ( $R$ is the number of channels in the correlator device), counting respectively $n_{1}, n_{2}, \ldots, n_{M+R}$ photons in each of them. During the first $M$ intervals the condition $n_{i}>k$ is fulfilled $t$ times. The signal in the $m$-th channel is given by

$$
S_{m}=\sum_{i=1}^{M}\left(n_{c}\right)_{i} n_{i+m}-\frac{t}{M} \sum_{i=1}^{M} n_{i}
$$

where $n_{c}$ is defined as in $\left(3^{\prime}\right)$.

In the case of $\langle n\rangle \beta \ll 1$, it is known that the statistical noise of the FCS photon detection process dominates $\left[{ }^{4}\right]$. The noise is defined as the square root of the dispersion of the signal (15) taking the quantities $n_{i}$ and $n_{i+m}$ for noncorrelated.

In the expression (15) $t$ members of type $\left(1-\frac{t}{M}\right) n_{k}$ and $(M-t)$ members of type $\left(-\frac{t}{M}\right) n_{k}$ occur (a stochastic nature of $t$ does not increase the noise). We obtain

$$
\operatorname{var} S_{\infty}=\frac{(M-t) t}{M} \operatorname{var} n \simeq \frac{(M-t) t}{M}\langle n\rangle .
$$

The signal-to-noise ratio only weakly depends on the choice of the clipping level $k$ in the region of $\langle n\rangle \pm(\operatorname{var} n)^{1 / 2}$. Choosing the point $k=$ $=\langle n\rangle$, most suitable for our calculations, we obtain from (15):

$$
S_{m}=(2 \pi)^{-1 / 2} \beta f(m T)\langle n\rangle^{3 / 2} M
$$

and from (16) $(t=M / 2)$ :

$$
\operatorname{var} S_{\infty}=\frac{1}{4} M\langle n\rangle
$$

So for the signal-to-noise ratio we obtain:

$$
\mu_{m} \equiv \frac{S_{m}}{\left(\operatorname{var} S_{\infty}\right)^{1 / 2}}=(2 / \pi)^{1 / 2} \sqrt{M} \beta\langle n\rangle f(m T) .
$$

This is only $(\pi / 2)^{1 / 2}$ times smaller than that of the nonclipped signal. The (insignificant) loss of information is due to the use of the clipping operation in the evaluation of the light intensity.

\section{Summary}

To obtain the same signal-to-noise ratio in an FCS experiment as in an PCS experiment, one needs significantly greater $\left(\beta^{-1 / 2}\right.$ times) counting speeds. One possibility to make operation at high speeds feasible is the use of the clipping operation. Both the form of the autocorrelation function of the fluorescence photon count and the signal-to-noise ratio are influenced by this. By measuring the clipped autocorrelation function 
instead of the nonclipped one in an FCS experiment, one loses only $(\pi / 2)^{1}{ }_{2}$ times in the signal-to-noise ratio if $\beta \ll \beta\langle n\rangle \ll 1$. Besides, the form of the signal remains undistorted.

The authors would like to thank Prof. E. Lippmaa for a number of discussions and J. Pelt for helpful criticism.

\section{Appendix: the relaxation of concentration fluctuations}

In an FCS experiment a certain kind of solute molecules in a well. defined open sample volume are continuously excited, and the intensity of the corresponding fluorescence is measured. Let the exciting intensity at point $r$ be $i(\boldsymbol{r})$. The instantaneous intensity of fluorescence can be expressed through the concentration of fluorescent molecules $C(\boldsymbol{r}, t)$ as

$$
I(t)=\int C(\boldsymbol{r}, t) i(\boldsymbol{r}) \mathrm{d} \boldsymbol{r} .
$$

Here we have neglected the intensity fluctuations caused by the random differences in the phases of different light beams. Being the object of study in PCS, these fluctuations are averaged out in FCS, because the detection area is much larger than the coherence area, and the counting interval is far longer than the coherence time.

The fluorescence intensity has the following dispersion:

$$
\operatorname{var} I(t)=\beta\langle I\rangle^{2},
$$

$\beta$ having the same order of magnitude as the reciprocal value of the number of molecules under observation $\left[{ }^{4}\right]$.

If one solute molecule is situated at point $\boldsymbol{r}$, the conditional probability density for the fluctuation of the fluorescence intensity at the initial moment $\delta I_{0}$, is a distribution with mean $i(\boldsymbol{r})$ and with dispersion $\beta\langle I\rangle^{2}$ :

$$
w\left(\delta I_{0} \mid \boldsymbol{r}\right)=\varphi\left(\frac{\delta I_{0}-i(\boldsymbol{r})}{\beta^{1 / 2}(I\rangle}\right) .
$$

In the special case $\beta \ll 1$

$$
i(\boldsymbol{r}) \ll\left|\delta I_{0}\right|
$$

and the distribution (A3) is nearly Gaussian, so that expanding $w\left(\delta I_{0} \mid \boldsymbol{r}\right)$ into a series, we obtain

$$
w\left(\delta I_{0} \mid \boldsymbol{r}\right) \simeq \varphi\left(\frac{\delta I_{0}}{\beta^{1 / 2}\langle I\rangle}\right)\left[1+\frac{\delta I_{0}}{\beta\langle I\rangle^{2}} i(\boldsymbol{r})\right] .
$$

The probability density $w\left(\delta I_{0} \boldsymbol{r}\right)$ as a function of the position $\boldsymbol{r}$ (the likelihood function) can be interpreted as a relative (unnormalized) probability density of the location of any molecule, or, in the case of ideal solutions, as a relative conditional expectation value of the concentration:

$$
E\left\{\delta C(\boldsymbol{r}) \mid \delta I_{0}\right\}=\delta I_{0} \frac{i(\boldsymbol{r})\langle C\rangle}{\beta\langle I\rangle^{2}} .
$$

It is noteworthy that the expectational value of the concentration fluctuations of a fluorescent (or, as can be shown, even any other nonfluorescent) component is proportional to the fluctuation of the fluorescence intensity.

The relaxation of the expectational values of concentrations in the 
ensemble of initial states $\delta I_{0}$ is expressed by the following system of kinetic equations:

$$
\frac{\partial x_{i}(\boldsymbol{r}, \tau)}{\partial \tau}=D_{i} \nabla^{2} x_{i}(\boldsymbol{r}, \tau)+\sum_{k} T_{i k} x_{k}(\boldsymbol{r}, \tau)
$$

with

$$
x_{i}(\boldsymbol{r}, \tau) \equiv E\left\{\delta C_{i}(\boldsymbol{r}, \tau) \mid \delta I(0)=\delta I_{0}\right\} .
$$

Here $D_{i}$ denotes the diffusion coefficient of the $i$-th component and $T_{i k}$ - the element of the matrix of rate constants of chemical reactions.

From the linearity of the system (A7) it follows that its solution at the initial condition (A6) is expressed as

$$
E\left\{\delta C_{i}(\boldsymbol{r}, \tau) \mid \delta I(0)=\delta I_{0}\right\}=\delta I_{0} f_{i}(\boldsymbol{r}, \tau),
$$

so that

$$
E\left\{\delta I(\tau) \mid \delta I(0)=\delta I_{0}\right\}=\delta I_{0} f(\tau) .
$$

This relation is convenient for deriving an expression for the clipped autocorrelation function.

\section{REFERENCES}

1. C a 11 en, H. B., We 1 t o n, T. A., Pliys. Rev., 83, 34 (1951).

2. E 1 s o n, E. L., M a g d e, D., Biopolymers, 13, 1 (1974).

3. Mag d e, D., E 1 s o n, E. L., W e b b, W. W., Biopolymers, 13, 29 (1974).

4. Ko p p e 1, D. E, Phys. Rev., A10, 1938 (1974).

5. Ed. Cum mi n s, H. Z., P ike, E. R., Photon Correlation and Light Beating Spectroscopy, NATO Advanced Study Institute Series, Series B: Physics, 3, New York, London, 1974.

6. J a keman, E., Pike, E. R., J. Phys., A2, 411 (1969).

7. S a 1 e h, B. E. A., Hen d r ix, J., J. Phys., A8, 1134 (1975).

8. Crooker, P. P., Hoover, J. A., Rev. Sci. Instrum., 47, 574 (1976).

Academy of Sciences of the Estonian SSR, Institute of Cybernetics

Received

Nov. 5, 1976

P. KASK, T. KANDLER

\section{CLIPPING-OPERATSIOONI MÕJU FLUORESTSENTSI KORRELATSIOONSPEKTROSKOOPIAS}

Käsitletakse footonite korrelatsioonspektroskoopias laialdaselt kasutatava nn. clippingehk pügamisoperatsiooni rakendamist fluorestsentsi korrelatsioonspektroskoopias. Clipping moonutab autokorrelatsioonifunktsiooni kuju üksnes lineaarselt ning vähendab signaalimüra suhet $(\pi / 2)^{1 / 2}$ korda.

\section{П. КАСК, Т. КЯНДЛЕР}

\section{ПРИВЯЗКА В КОРРЕЛЯЦИОННОЙ СПЕКТРОСКОПИИ ФЛУОРЕСЦЕНЦИИ}

Обсуждается влияние привязки, стандартной операции в корреляционной спектроскопии фотонов, применительно к корреляционной спектроскопии флуоресценции. Показано, что операция привязки в одном канале искажает автокорреляционную функцию линейно и уменьшает отношение сигнала к шуму в $(\pi / 2)^{1 / 2}$ раз. 